\title{
Biophysical and Physiological Properties of Synthetic Surfactants Containing a Surfactant Protein Analog
}

\author{
Shoichi Chida \\ Department of Pediatrics, \\ Iwate Medical University School of Medicine, Morioka, Japan
}

\begin{abstract}
Purpose: The in vitro biophysical and in vivo physiological properties of two synthetic surfactants containing either an SP-B or SP-C analog were compared with those of a modified bovine surfactant preparation, Surfactant-TA (Surfacten).

Methods: Biophysical properties were measured using a modified Wilhelmy surface balance with reference to adsorption, spreading, minimum and maximum surface tension, and $\%$ trough area at $10 \mathrm{mN} / \mathrm{m}$. Physiological properties were measured by recording pressure-volume curves using rabbit fetuses delivered prematurely. Rabbit fetuses were randomly assigned to receive one of the surfactants intratracheally. Premature littermates and term fetuses were used as controls.

Results: The SP-C lipid mixture and Surfacten exhibited similar biophysical and physiological properties appropriate for a pulmonary surfactant, whereas the SP-B lipid mixture did not satisfy these criteria.

Conclusions: The SP-C lipid mixture exhibited properties appropriate for a pulmonary surfactant, comparable to Surfacten. In contrast, the SP-B analog may not be an alternative to natural SP-B. Based on our study, clinical trials of surfactant containing the SP-C analog can be justified.
\end{abstract}

Keywords: respiratory distress syndrome, surfactant protein$\mathrm{B}$, surfactant protein-C

\section{Introduction}

Fujiwara and coworkers (1) reported in 1980 that a modified bovine surfactant demonstrated remarkable improvements in 10 preterm infants with severe respiratory distress syndrome (RDS). This discovery led to the development of animal-derived or synthetic surfactant preparations that have been validated using randomized trials. Most current products for treating RDS use biological surfactants, as they are universally accepted and credited with improving overall mortality. However, there are concerns that animal-derived products could transmit infectious agents, and they are relatively expensive.

Biological surfactants consist mainly of lipids and 1-2\% of hydrophobic surfactant protein-B (SP-B) and SP-C. In contrast, first-generation synthetic surfactants contain no surfactant proteins. Tanaka et al. found that four components, namely dipalmitoyl phosphatidylcholine (DPPC), phosphatidyl glycerol (PG), palmitic acid (PA), and hydrophobic surfactant proteins are essential for optimal surface activity (2). Knowledge of the functional significance of hydrophobic surfactant proteins, as well as progress in molecular technology, has allowed the production of surfactant protein analogs. This paper presents the biophysical and physiological properties of two synthetic surfactants containing either an SP-B or SP-C analog, and of a modified bovine surfactant preparation. 


\section{Materials and Methods}

\section{Synthetic surfactants}

Human SP-B comprises 79 amino acid residues in a periodic hydrophilic/hydrophobic pattern. KL4, developed by Cochrane and Revak $(3,4)$, is a 21 residue peptide designed to mimic the periodic pattern of the N-terminal part of human SP-B using a repeat sequence of four leucine (L) residues sepa- rated by individual lysine $(\mathrm{K})$ residues (Table 1$)$. Human SP-C comprises 35 residues. SP-C leucine 16 is a 23-residue peptide that was designed to mimic the very hydrophobic nature of SP-C using a sequence of 16 leucines in the C-terminal half of the peptide (Table 1). Both peptide analogs were produced by peptide synthesis.

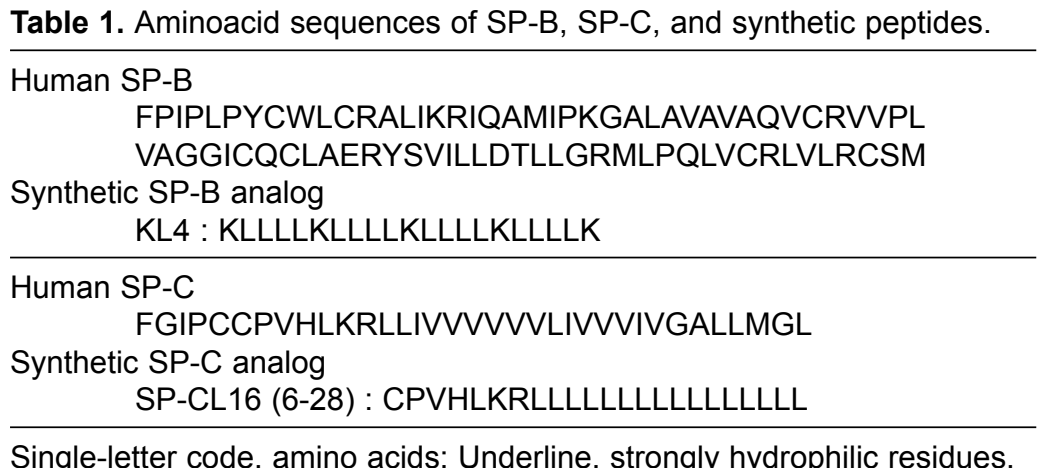

Synthetic surfactants were prepared using DPPC, palmitoyl oleoyl phosphatidylglycerol (POPG) or PG, PA, and KL4 or SP-C leucine 16, according to the original publications (3-5) (Table 2). The mixtures were sonicated before use.

Table 2. Synthetic surfactants containing the SP-B or SP-C analog.

$\begin{aligned} & \text { SP-B lipid mixture } \\ & \text { DPPC : POPG : PA : SP-B analog }=75: 25: 15: 3(\mathrm{w} / \mathrm{w}) \\ & (\mathrm{KL} 4)\end{aligned}$
$\begin{aligned} & \text { SP-C lipid mixture } \\ & \text { DPPC : PG : PA : SP-C analog }=75: 25: 10: 3(\mathrm{w} / \mathrm{w}) \\ & \quad(\text { SP-CL16) }\end{aligned}$
$\begin{aligned} & \text { DPPC, dipalmitoyl phosphatidylcholine; POPG, palmitoyl oleoyl } \\ & \text { phosphatidylglycerol; PG, phosphatidylglycerol; PA, palmitic acid. }\end{aligned}$

Measurement of biophysical and physiological properties of the surfactants

The in vitro biophysical and in vivo physiological properties of two synthetic surfactants, SP-B and SP-C lipid mixtures, were compared with those of a modified bovine surfactant preparation, Surfactant-TA (Surfacten, Mitsubishi Tanabe Pharma Corp., Osaka, Japan). Biophysical properties were measured using a modified Wilhelmy surface balance with reference to adsorption, spreading, minimum and maximum surface tension, and $\%$ trough area at 10 $\mathrm{mN} / \mathrm{m}$ (Figure 1). Adsorption measures the change in surface tension when surfactant $(62.5 \mu \mathrm{g} / \mathrm{ml})$ is placed at the bottom of the container, whereas spread-
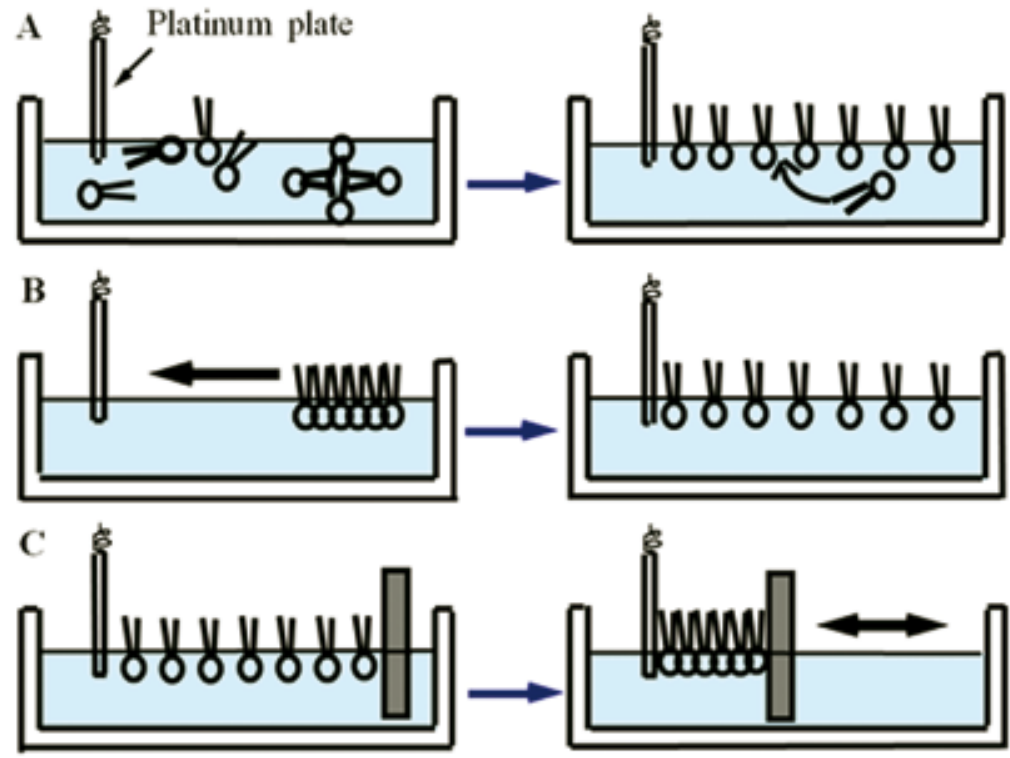

Figure 1. Measurement of the biophysical properties of a surfactant using a modified Wilhelmy surface balance.

A, adsorption; B, spreading; C, dynamic surface tension-area diagram. 
ing measures the change in surface tension when surfactant $\left(1.5 \mu \mathrm{g} / \mathrm{cm}^{2}\right)$ is placed on the surface of a layer of saline in a container; importantly, the volume of surfactant added is just sufficient to form a monolayer on the saline surface. Following these measurements, the surface film is compressed and expanded using a barrier to directly record a dynamic surface tension-area diagram. The minimum and maximum surface tension and \% trough area at $10 \mathrm{mN} / \mathrm{m}$ were obtained from the diagram. Physiological properties were measured by record- ing pressure-volume curves using the apparatus shown in Figure 2. Rabbit fetuses were delivered prematurely and randomly assigned to receive one of the surfactants intratracheally at a dose of 50 or $100 \mathrm{mg} / \mathrm{kg}$, then the tracheomized fetuses were placed in a plastic box and connected to a tube. Premature littermates and term fetuses were used as controls.

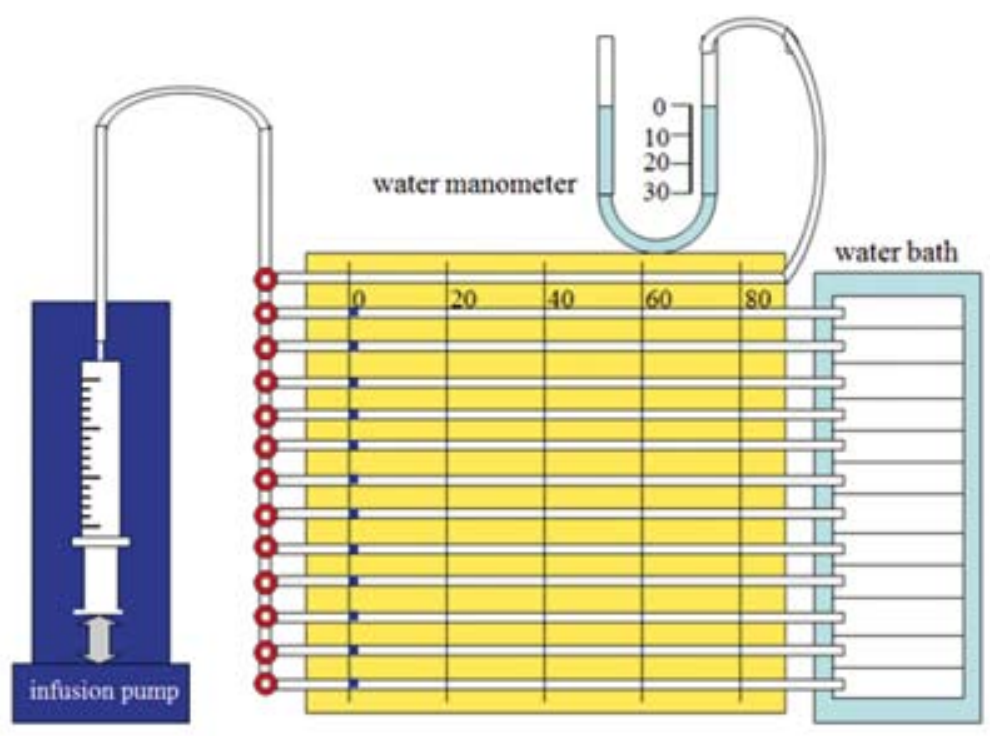

Figure 2. Measurement of the physiological properties of a surfactant

\section{Lung morphology}

The pulmonary pressure-volume relationship indicates the volume of air retained in the lung at a given pressure. However, since this volume may be affected by entrapped air or an uneven distribution of air in the lung, alveolar expansion and alveolar homogeneity were assessed using lung sections.

Lung morphology was compared in rabbit fetuses treated with either the SP-B or SP-C lipid mixture, or with Surfacten, at a deflation pressure of $10 \mathrm{cmH}_{2} \mathrm{O}$. Alveolar expansion was measured as the ratio of alveolar field to lung area using a computer-aided image analyzer. Alveolar homogeneity was graded based on the rate of alveolar homogeneity from zero (no homogeneity) to 5 (all alveoli expanded homogeneously) for every $25 \%$ increase of homogeneity.

\section{Electron microscopic visualization of the surfac- tants}

Macromolecular assemblies of these surfactants were visualized using electron microscopy.

\section{Results and Discussion}

Surfacten exhibits biophysical and physiological properties appropriate for a pulmonary surfactant: 1) rapid adsorption, rapid spreading, and low minimum surface tension; 2) conversion of the pressure-volume characteristics of a premature animal lung to those of a mature lung; 3) homogeneous alveolar expansion at low transpulmonary pressure (6). Therefore, the surface-active properties of the SP-B and SP-C lipid mixtures were compared with those of Surfacten.

\section{Measurement of biophysical and physiological properties of the surfactants}

The surface tension of the SP-B lipid mixture was significantly higher than that of the SP-C lipid mixture and Surfacten (Figures 3A and 3B). The SP-C lipid mixture and Surfacten rapidly adsorbed and spread. The dynamic surface tension-area diagram showed that the surface tension of the SP-B lipid mixture reached $10 \mathrm{mN} / \mathrm{m}$ with $55 \%$ surface compression, which is a rather large surface com- 
pression, and that the maximum surface tension of the SP-B lipid mixture was significantly higher than that of the SP-C lipid mixture and Surfacten (Figure 3C). In contrast, the SP-C lipid mixture and Surfacten exhibited low surface tension with only $20 \%$ surface compression and low maximum surface tension.

A

B

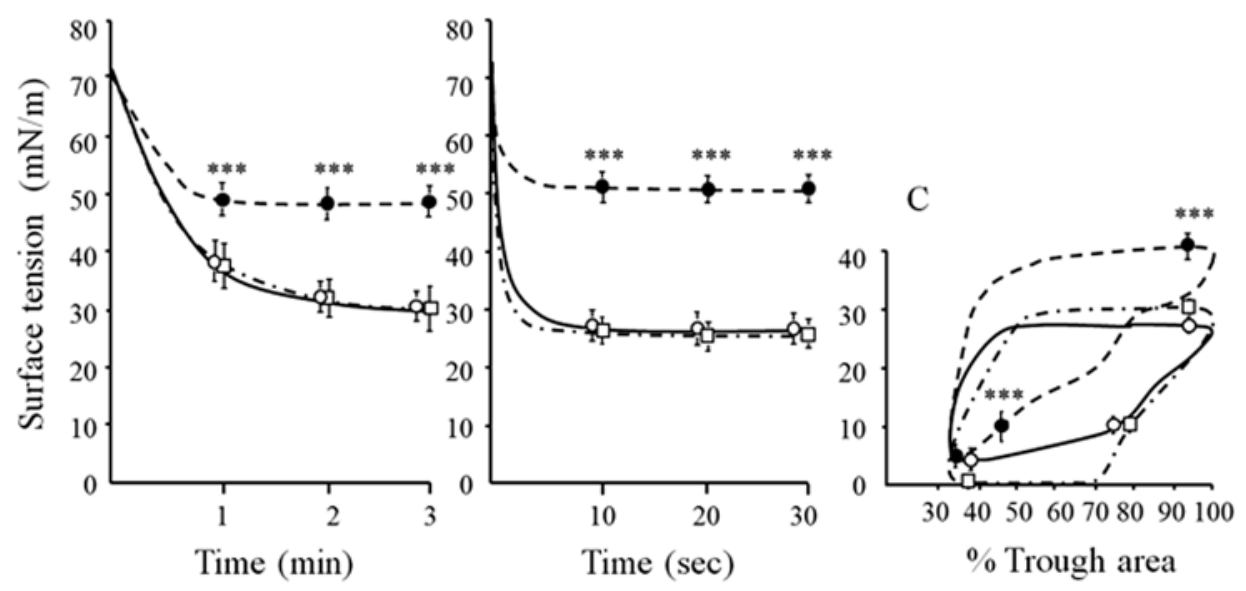

Figure 3. Biophysical properties of the surfactants.

$A$, adsorption; $B$, spreading; $C$, dynamic surface tension-area diagram.

Surfacten; SP-B lipid mixture; SP-C lipid mixture $(n=10$, mean $\pm S D)$.

${ }^{* * *} p<0.001$.

These results indicate that the SP-C lipid mixture and Surfacten exhibited similar biophysical properties and have in vitro surface-active properties appropriate for a pulmonary surfactant, whereas the biophysical properties of the SP-B lipid mixture do not satisfy these criteria.
Furthermore, the SP-C lipid mixture and Surfacten improved lung volume to mature levels at $50 \mathrm{mg} / \mathrm{kg}$ when administered to rabbit fetuses (Figures 4A and 4B), whereas the SP-B lipid mixture failed to improve lung volume to mature levels at a dose of $100 \mathrm{mg} / \mathrm{kg}$ (Figures 4A and 4C).

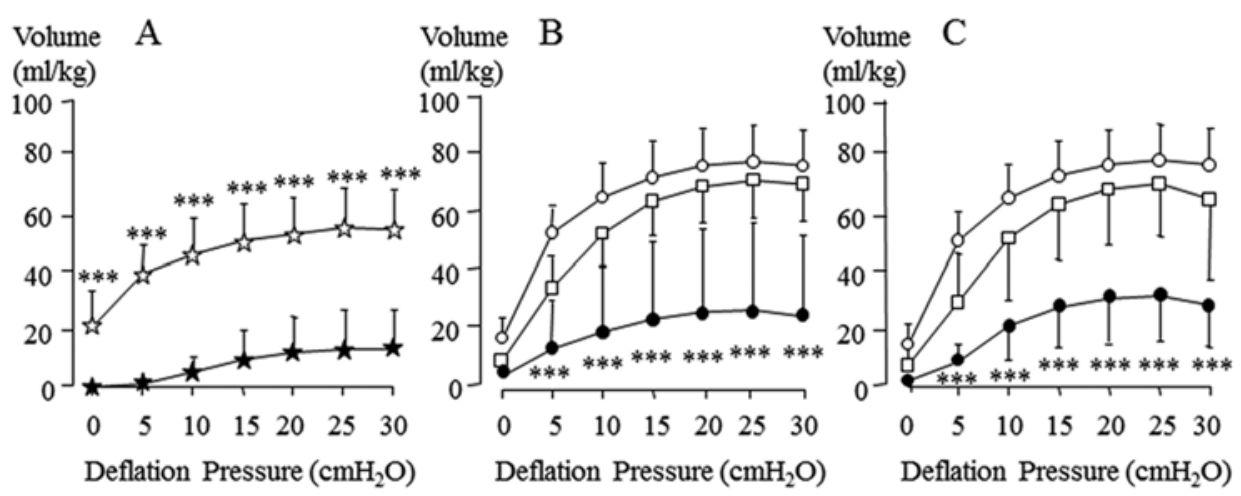

Figure 4. Lung volume of rabbit fetuses treated with the surfactants.

A, controls; B, fetuses treated with $50 \mathrm{mg} / \mathrm{kg}$ of the surfactants; C, fetuses treated with $100 \mathrm{mg} / \mathrm{kg}$ of the surfactants. premature control; mature control; Surfacten;

SP-B lipid mixture; SP-C lipid mixture ( $n=7-13$, mean $\pm S D)$. ${ }^{* * *} p<0.001$.

\section{Lung morphology}

The alveoli of premature lungs treated with $50 \mathrm{mg} /$ $\mathrm{kg}$ of the SP-B lipid mixture failed to show expansion to mature levels (Figures 5B and 6A), whereas premature lungs treated with the SP-C lipid mixture or Surfacten reached mature levels at a dose of 50 $\mathrm{mg} / \mathrm{kg}$ (Figures 5B, 5C and 6C). Alveolar expansion following treatment with $100 \mathrm{mg} / \mathrm{kg}$ did not differ significantly among the three surfactant groups (Figure 7A), but the SP-B lipid mixture provided a significantly smaller area of homogeneous alveolar expansion than did the mature control (Figure 7B). 
Figure 5. Lung histology of rabbit fetuses treated with the surfactants.

A, premature control;

$\mathrm{B}$, mature control;

C, Surfacten $50 \mathrm{mg} / \mathrm{kg}$;

D, Surfacten $100 \mathrm{mg} / \mathrm{kg}$.
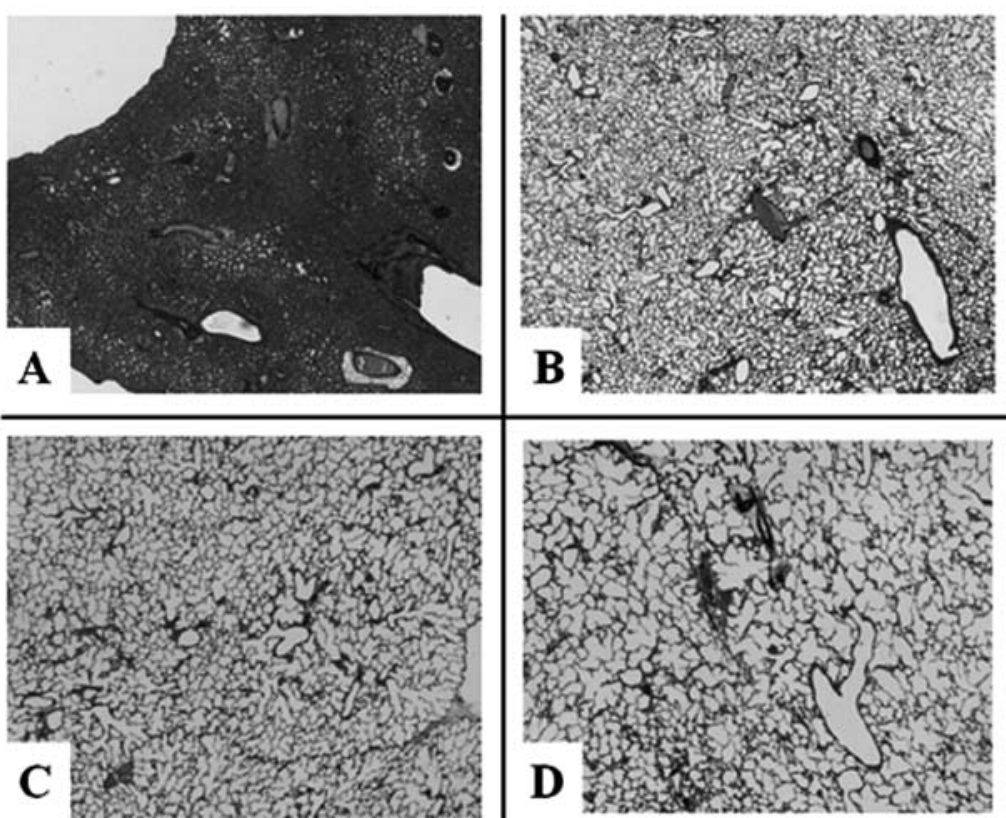
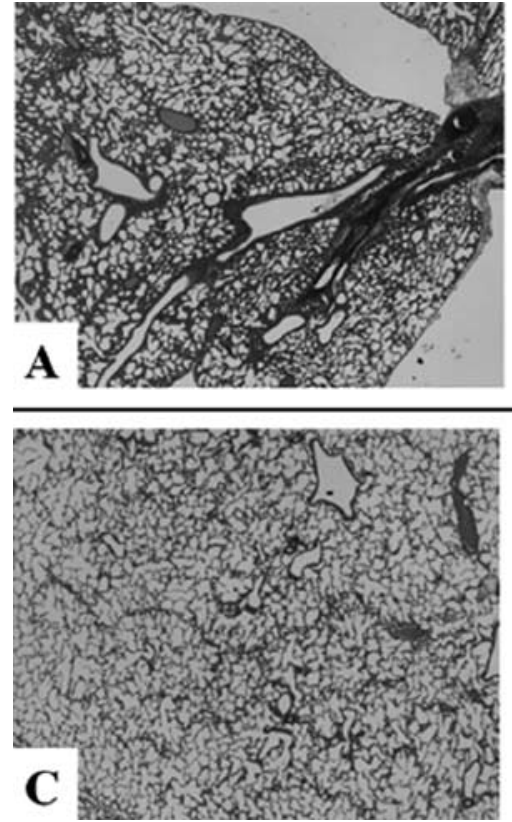
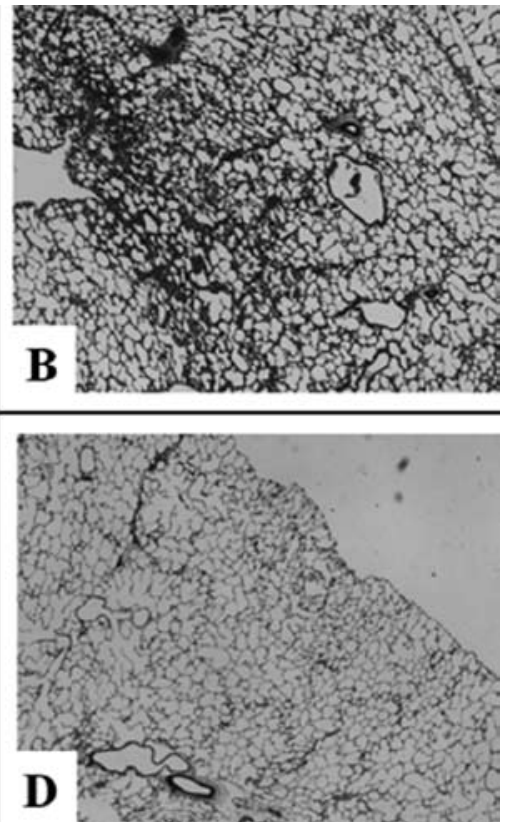

Figure 6. Lung histology of rabbit fetuses treated with the surfactants. A, SP-B lipid mixture $50 \mathrm{mg} / \mathrm{kg}$;

B, SP-B lipid mixture $100 \mathrm{mg} / \mathrm{kg}$;

C, SP-C lipid mixture $50 \mathrm{mg} / \mathrm{kg}$;

D, SP-C lipid mixture $100 \mathrm{mg} / \mathrm{kg}$.
Figure 7. Alveolar expansion and alveolar homogeneity of rabbit fetus lungs treated with the surfactants.

$\mathrm{M}$, mature control;

$\mathrm{P}$, premature control;

St, Surfacten;

B, SP-B lipid mixture;

C, SP-C lipid mixture

$(n=4-8$, mean $+S D)$.

** $p<0.01$;

*** $p<0.001$.

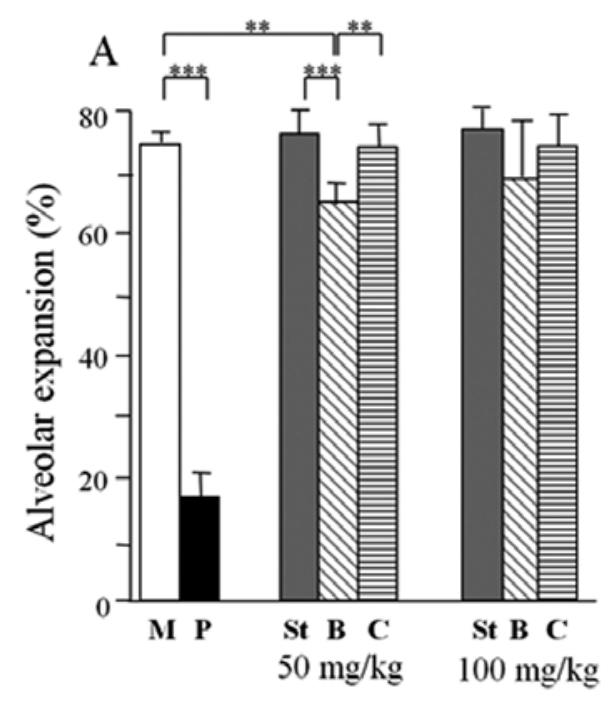




\section{Electron microscopic visualization of the surfac-} tants

All surfactants self-assembled to provide myelin-like structures consisting of electron-dense and lucent multi-lamella with an approximately $50 \AA$ periodicity (Figure 8 ). Surfacten demonstrated long rod-like lamellar structures (Figure 9A). The SP-B and SP-C lipid mixtures also assembled into rod-like lamellar structures (Figures 9B and 9C), but the rods formed by the SP-C lipid mixture were thinner and shorter than the other two surfactants. It is generally accepted that at the air-liquid interface, SP-B acts as a surfaceseeking peptide and cross-links two lipid bilayers, whereas SP-C functions as a transmembrane peptide and spans DPPC. The observed heterogeneity of the surfactant structures can thus be ascribed to these differences in properties of the surfactant protein analogs, the lipid constituents, and/or the use of sonication (SP-B and SP-C lipid mixtures).

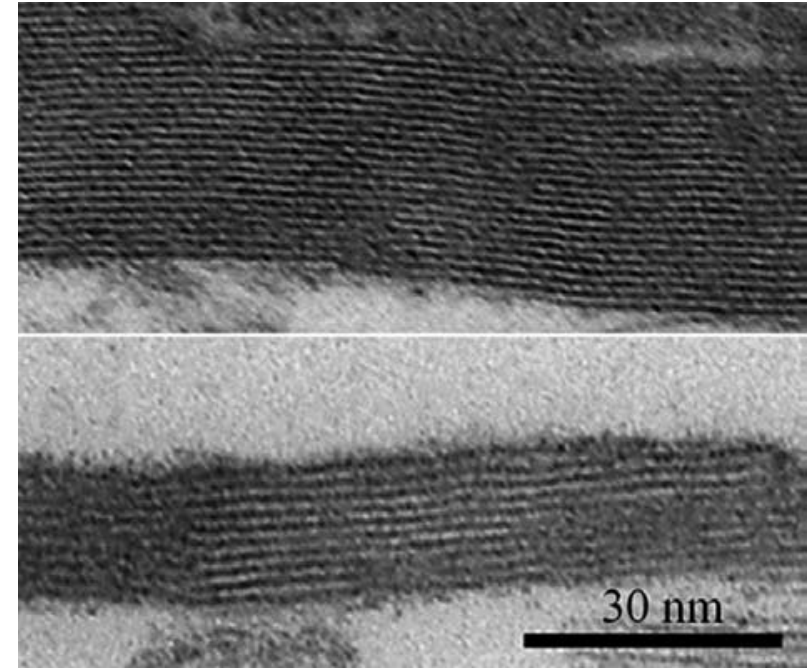

Figure 8. Electron microscopic images of the surfactants. Upper panel, Surfacten; Lower panel, SP-C lipid mixture.

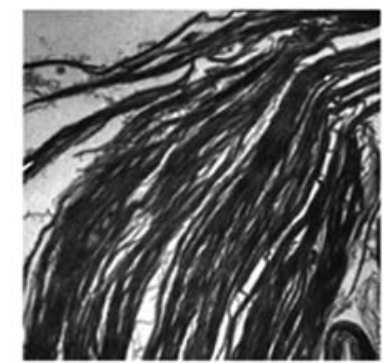

A

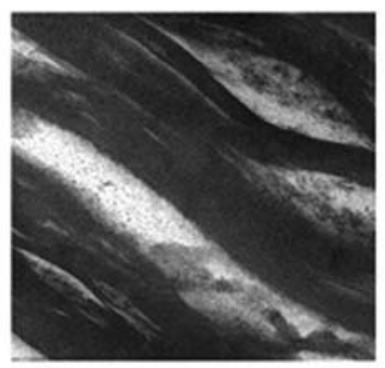

A

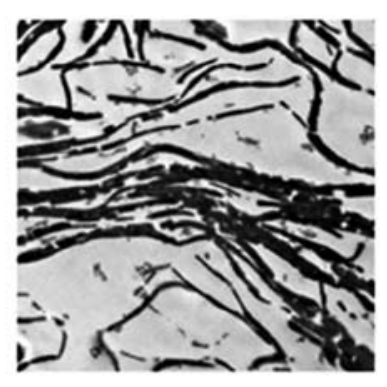

B

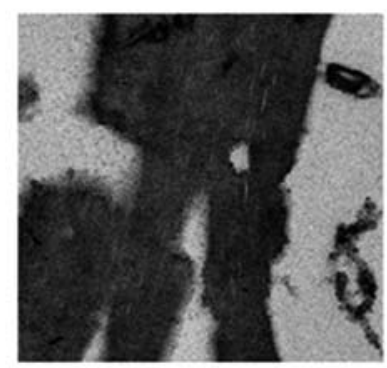

B
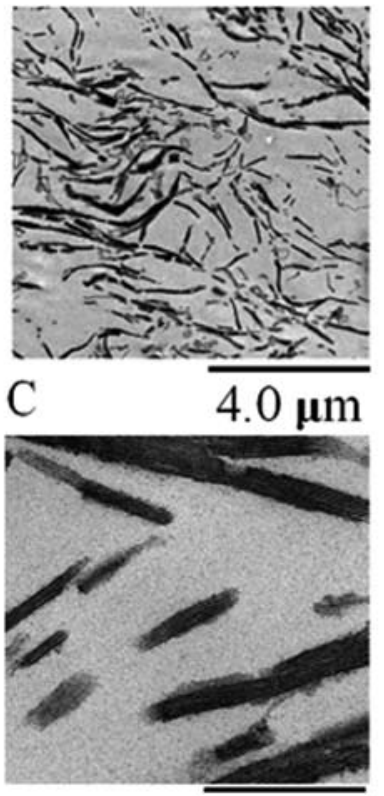

C

$200 \mathrm{~nm}$

Figure 9. Electron microscopic images of the surfactants.

A, Surfacten; B, SP-B lipid mixture; C, SP-C lipid mixture.

\section{Conclusions}

The SP-C lipid mixture exhibited properties appropriate for a pulmonary surfactant, comparable to Surfacten. In contrast, the SP-B analog may not be an alternative to natural SP-B. Based on our study, clinical trials of surfactant containing the SP-C analog can be justified. A synthetic surfactant con- taining the SP-B analog has recently been approved for the prevention of RDS in premature infants in the United States, because two multinational phase 3 trials showed its safety and efficacy to be similar to that of biological surfactants (Survanta and Curosurf). 


\section{References}

(1) Fujiwara, T., Maeta, H., Chida, S., et al. Artificial surfactant therapy in hyaline-membrane disease. Lancet, i (8159): 55-59, 1980.

(2) Tanaka, Y., Takei, T., Aiba, T., et al. Development of synthetic lung surfactants. J Lipid Res, 27: 475-485, 1986.

(3) Cochrane, CG. and Revak, SD. Pulmonary surfactant protein B (SP-B): structure-function relationships. Science, 254: 566-568, 1991.

(4) Cochrane, CG., Revak, SD., Merritt, TA, et al. The efficacy and safety of $\mathrm{KL}_{4}$-surfactant in preterm infants with respiratory distress syndrome. Am J Respir Crit Care Med, 153: 404-410, 1996.

(5) Takei, T., Hashimoto, Y., Ohtsubo, E., et al. Characterization of poly-leucine substituted analogues of the human surfactant protein SP-C. Biol Pharm Bull, 19: 1550-1555, 1996.

(6) Fujiwara, T. Surfactant Replacement in Neonatal RDS. In: Robertson B, Van Golde LMG, Batenburg J (eds), Pulmonary Surfactant. Elsevier, Amsterdam, pp 479-503, 1984.

\section{Shoichi Chida}

19-1 Uchimaru, Morioka 020-8505, Japan

$\mathrm{Tel}:+81-196-651-5111$

Fax: +81-196-651-0515

Email address: schida@iwate-med.ac.jp 